\title{
Asymmetry in 3-D perceptual organization: Ground-like surface superior to ceiling-like surface
}

\author{
JASON S. MCCARLEY and ZIJIANG J. HE \\ University of Louisville, Louisville, Kentucky
}

\begin{abstract}
Attneave (1954) and Barlow (1961) proposed that the visual system might increase efficiency of representation by preferentially encoding spatiotemporally redundant patterns of the external world. The present experiments tested the application of this principle to three-dimensional (3-D) perceptual organization, capitalizing on the ecological constraint that human observers must frequently interact with objects arranged on the ground or on a surface parallel to it (Gibson, 1950). Observers performed a task that required them to perceptually segregate and search multiple items distributed in depth and embedded within a larger, 3-D array of distractors. Stimulus displays were organized to globally recede top-away in depth, as if attached to an underlying ground-like surface, or bottom-away, as if attached to an overhanging ceiling-like surface; ground-like and ceiling-like displays differed only in the direction of disparity gradient within the displays. Primary findings revealed superior performance with ground-like displays, suggesting that spatially and stereoscopically distributed items are more easily organized to represent an ecologically representative pattern, even when no inherent physical regularities favor that pattern.
\end{abstract}

The visual system is charged with the task of representing the external world in a format suitable for object recognition and the control of behavior (Marr, 1982). This process begins with the formation of the retinal image, and much of the subsequent peripheral and early cortical image processing within the visual system is relatively well understood (e.g., DeValois \& DeValois, 1988). Accumulating computational and experimental evidence, however, suggests that many of the perceptual processes underlying visual behavior and phenomenology may not be directly mediated by filtering or some other treatment of the retinal image itself. Rather, the immediate processing of image features seems to give way to a higher level visual description, preceding the first stages of visual awareness, in which the properties of distal surfaces are explicitly represented (e.g., the $21 / 2-D$ sketch [Marr, 1982] and the visual surface representation [Gibson, 195v; Grossberg \& Mingolla, 1985; Nakayama, He, \& Shimojo, 1995; Ramachandran \& Cavanagh, 1985]). Thus, the mechanisms underlying visual search (Aks \& Enns, 1996; Enns \& Rensink, 1990; He \& Nakayama, 1992), texture segregation (He \& Nakayama, 1994b),

Some of these results were presented at the 1996 Annual Meeting of the Psychonomic Society. This research was supported by a Sigma Xi Grant-in-Aid of Research to J.S.M., by a Sloan Research Fellowship to Z.J.H, and by ONR Grant 994984 . The authors thank G. John Andersen, Paul Atchley, Thomas Sanocki, and an anonymous reviewer for their helpful comments on an earlier draft of the article. Correspondence concerning this article should be addressed to Z. J. He, Department of Psychology, University of Louisville, Louisville, KY 40292 (e-mail: z0he0002@athena.louisville.edu). motion perception (He \& Nakayama, 1994a; Shimojo \& Nakayama, 1990), and Gestalt grouping (Palmer, Neff, \& Beck, 1996; Rock \& Brosgole, 1964)-processes that have been taken to tap the most rudimentary levels of visual phenomenology-seem to have little or no access to the unelaborated features of the retinal image but, apparently, proceed from a representation in which amodal shape and three-dimensional (3-D) surface layout have been achieved.

A likely benefit of a visual surface representation is more efficient coding, through which multiple, spatially separated features are encoded as unitary, spatially extensive structures. Such recoding is possible because the stimuli to be represented are not arbitrary or capricious but redundant and highly predictable; of the enormous set of patterns that might be projected to the retinas, only a small subset is likely to be encountered in an organism's natural environment (Attneave, 1954; Barlow, 1961). Capitalizing on this redundancy, the visual system may encode the behaviorally relevant patterns or arrangements in which stimulus features occur, rather than disorganized arrays of the disjointed features themselves. Economy of representation is maximized when the most frequently encoded patterns of stimulation are represented with the fewest possible signals. Thus, an efficient perceptual system will most readily represent environmentally pervasive and behaviorally relevant external patterns (Barlow, 1961). This principle may also provide the neural basis of Helmholtz's (1910/1962) likelihood principle, which dictates that an adaptive visual system should perceive the external arrangement most likely to have cast the present retinal image (Pomerantz \& Kubovy, 1986). 
These considerations suggest that a recurrent or ecologically pervasive stimulus pattern will be coded and perceived more easily than a similar but less ecologically representative configuration. The ecological pervasiveness of gravity and of the terrestrial surface-of-support may provide a ready environmental characteristic to be so exploited; with fairly infrequent exceptions, objects not in direct contact with the ground must find support from a surface roughly parallel to it (Gibson, 1950, 1979; Sedgwick, 1983). This ecological regularity-along with the laws of perspective projection, which dictate that a surface parallel to the horizon and below eye height must recede in depth with height in the field -imposes order on the structure of the perceptual world (Sedgwick, 1986). Because the ground on which an observer stands is necessarily below eye height, objects attached to the same ground will generally be placed higher in the field of view as they are situated farther in depth. Moreover, the secondary supporting surfaces surrounding an observerdesktops and countertops, for example - are ordinarily placed below eye height as well, so that the objects on them may be more easily seen and grasped. Even indoors, therefore, where an observer is enclosed by walls perpendicular to the ground and by a ceiling overhead, the majority of behaviorally relevant objects and the surfaces on which they are arrayed will tend to recede in depth with height in the visual field. Thus, spatial arrangements in which objects farther from an observer are placed higher in the visual field, although not universal within the visual environment of humans, are frequent, if not predominant. Perhaps more important, they are often formed by objects that must guide behavior - those objects among and around which an observer must search, navigate, and reach, for example. Because this arrangement of stimuli contains no inherent physical regularities that make it less redundant than a similar but inverted arrangement of items attached to an overhanging surface, a wholly general purpose system of representation would not be expected to encode a display of items arrayed top-away in depth with any special efficiency. A perceptual system adapted through evolution or learning to operate efficiently within the visual environment of humans, however, could achieve economy of representation by preferentially encoding the recurrent stimulus structure imposed by the demand for underlying support. Perceptual organization might, therefore, be expected to favor such structure. The present experiments tested this hypothesis, examining the prediction that visual grouping and segregation will be more efficient within a ground-like arrangement of stimuli, in which stimuli are placed farther in distance as they are placed higher in the field of view, than within an inverse, ceiling-like arrangement.

\section{EXPERIMENT 1}

Experiment 1 tested the ability of participants to group and search along implicit planes differently oriented in 3-D space relevant to an observer, using task and stimuli similar to those of He and Nakayama (1995). Individual stimulus elements were arranged to form a vertical stack of three implicit horizontal planes, receding top-away to form ground-like displays or bottom-away to form ceiling-like displays (Figure 1). The participants were asked to search the middle implicit plane of elements and to indicate the presence or absence of an odd-colored target therein. If an ecologically relevant stimulus arrangement is indeed more easily organized perceptually, performance should be superior for search within ground-like displays.

\section{Method}

Observers. The participants were 5 men and 3 women, 1941 years of age, all of whom were experienced psychophysical observers. All the participants had normal or corrected-to-normal visual acuity, normal color vision, and normal stereopsis. One observer was aware of the experimental hypothesis, whereas the rest were naive.

Apparatus and Stimuli. The stimuli were presented on the monitor of a Commodore Amiga 2000 computer, with a frame rate of $60 \mathrm{~Hz}$, and were viewed through haploscopic glasses. Viewing distance was approximately $80 \mathrm{~cm}$. The participants were free to move their heads.

The stimuli were similar to those described in Experiment $2 \mathrm{~A}$ of He and Nakayama (1995) and are schematically depicted in Figure 1. Each display comprised a stereoscopically created $4 \times 3 \times$ 3 array of rectangular orange and green elements, displayed against a black background. The elements were stereoscopically slanted and arranged to form three horizontal arrays of coplanar elements, stacked vertically and receding in depth. Each horizontal array comprised three rows of four elements, with the middle row of each array centered at zero disparity. Elements were arranged so that, from the top of the display, Rows 1,2 , and 4 formed a single horizontal array, Rows 3, 5, and 7 another, and Rows 6, 8, and 9 the third. Thus, rows of elements belonging to different horizontal arrays were interlaced from top to bottom of the display, and the three implicit planes could only be segregated stereoscopically. Successive horizontal arrays were separated vertically by $107 \mathrm{~min}$ of arc. Successive rows within a horizontal array were separated by a center-tocenter vertical distance of $47 \mathrm{~min}$ of arc and by a center-to-center disparity of $22 \mathrm{~min}$ of arc. Within a row, the horizontal separation between elements varied randomly from 93 to $108 \mathrm{~min}$ of arc. The individual stimulus elements presented to one eye were rectangles of $54 \times 14$ min of arc, whereas those presented to the other eye were of approximately the same dimensions but were sheared to create stereoscopic slant of 6 min of arc from the top to the bottom of the element. Within ground-like displays, the implicit planes formed by horizontal arrays of elements slanted top-away in depth, so that the top row within the plane was of uncrossed disparity, the center row was centered at disparity, and the bottom row was of crossed disparity. Within ceiling-like displays, implicit planes slanted bottomaway in depth. The top and bottom horizontal arrays within each display were always considered distractors, and the target of the search, when present, was located within the middle horizontal array of elements. The target was either orange or green, whereas the rest of the elements in the middle horizontal array were of the alternative color (i.e., green or orange, respectively). In each of the top and bottom arrays, there were two, four, or six elements of the same color as the target, whereas the rest were of the alternative color. The display in the target-absent trials was the same as that in the target-present trials, except that elements in the middle horizontal array were all of the same color.

A fixation cross of no disparity appeared between trials, centered at the same location as the search display. 
A.
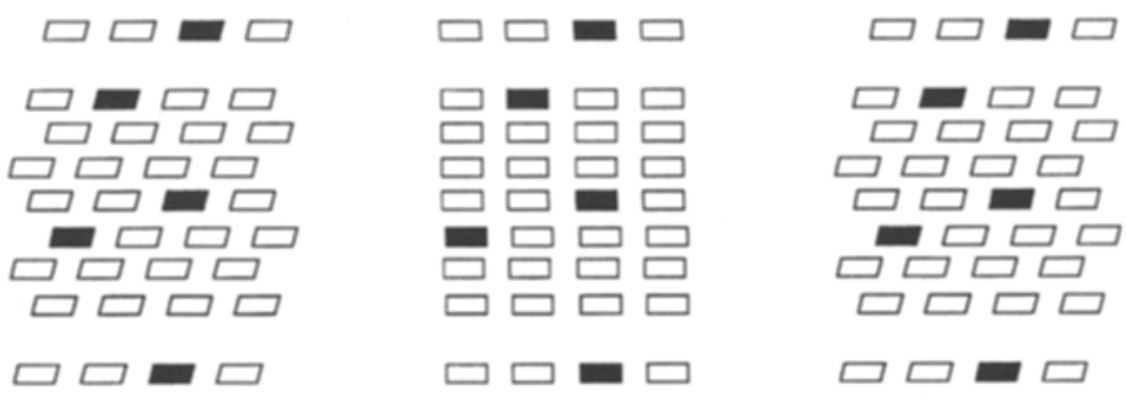

B.
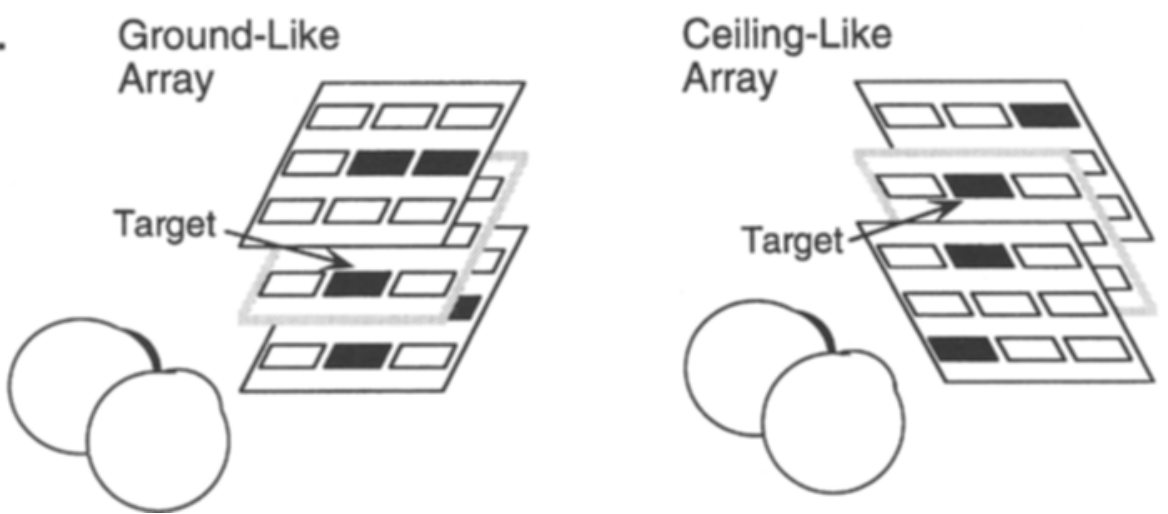

Figure 1. A: stereograms depicting stimuli like those used in Experiment 1. For all stereograms, cross-fusing the left and center images or parallel-fusing the center and right images will produce a ground-like display; cross-fusing the center and right images or parallel-fusing the left and center images will produce a ceiling-like display. B: a schematic illustration of the stimuli used in Experiment 1 . The observer's task was to search for an odd-colored element within the middle horizontal array, which is here denoted by a bold rectangle.

Procedure. The observers initiated each trial with a keypress. After a variable delay of 500-1,000 msec, the search display appeared. The orientation of the search display (ground-like or ceilinglike), the number of target-colored items within the display $(4,8$, or 12 ), and the presence or absence of the target varied randomly from trial to trial, under the constraint that all combinations of these conditions appear an equal number of times. The participants' task was to search the middle horizontal array of stimulus elements and to indicate with a keypress the presence or absence of an element differing in color from the others within that array. The participants were told that their reaction times (RTs) would be measured and that they should, therefore, respond as quickly as possible while maintaining accuracy. After conducting a short block of practice trials, each participant completed a total of 600 data collection trials, 50 target-present and 50 target-absent trials for each combination of stimulus orientation and number of target-colored distractors.

\section{Results and Discussion}

Mean RTs and error rates for search within groundlike and ceiling-like displays trials are presented in Figure 2. RTs three standard deviations or more beyond the mean of each cell and RTs for incorrect responses were excluded from the analysis. RTs and error rates were submitted to $2 \times 2 \times 3$ analyses of varaince (ANOVAs), with display orientation (ground-like or ceiling-like), target presence or absence, and number of target-colored distractors $(4,8$, or 12$)$ as factors. Of most immediate interest, RTs were consistently shorter within ground-like displays $[F(1,7)=14.11, p=.007]$, showing a mean advantage of $198.61 \mathrm{msec}(S E=52.88)$. The data were thus consistent with the proposition that a more ecologically relevant stimulus arrangement should have enabled better performance. As is typical of visual search data, response latencies were generally longer for target-absent than for target-present responses $[F(1,7)=23.78, p=.002]$, and consistent with the report of He and Nakayama (1995), the number of target-colored distractors within a display affected RTs modestly, producing a main effect of marginal significance $[F(2,14)=3.13, p=.075]$. Increases in RT with the number of target-colored distractors, however, were approximately the same within ground-like displays as within ceiling-like displays. Thus, differences in mean RT between ground-like and ceiling-like displays were fairly constant in magnitude as the number of targetcolored distractors within a display increased $(F<1)$, suggesting that performance with ceiling-like displays was no more susceptible to interference from target-colored distractors than was performance with ground-like displays. ${ }^{l}$ Additional interactions within RT data also fell short of significance. Within error rate data, only the main 

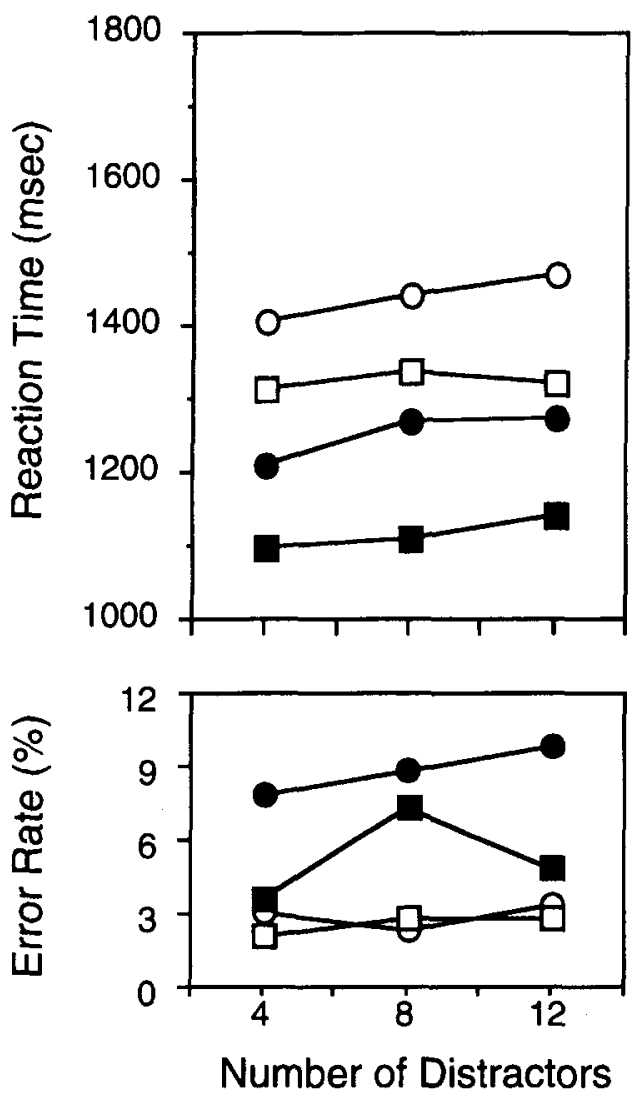

$\begin{array}{ll}\text { Target Absent: } & \text { Target Present: } \\ \text { O Ceiling-like } & \text { Ceiling-like } \\ \square \text { Ground-like } & \quad \text { Ground-like }\end{array}$

Figure 2. Reaction times and error rates for search of ceilinglike and ground-like displays in Experiment 1, as a function of the number of target-colored distractors within a display.

effect of target presence reached significance $[F(1,7)=$ $16.79, p=.005]$, indicating more accurate performance on target-absent trials. Error rates did not differ significantly between displays of opposite orientations $[F(1,7)=$ $3.75, p=.094$ ], but were generally lower for search within ground-like displays, indicating that differences in search time between displays of opposite orientations were not attributable to a speed-accuracy tradeoff.

\section{EXPERIMENT 2}

The results of Experiment 1 indicated, as was expected, superior performance for search within groundlike displays. The second experiment replicated and clarified this effect. Because the individual stimulus elements making up the displays of Experiment 1 were oriented differently in the ground-like and the ceiling-like displays, faster responses to ground-like displays might have resulted from potential anisotropies in processing local orientation in depth, rather than from asymmetries in establishing global organization of the oppositely tilting displays. Furthermore, Experiment 1 may have demonstrated only that coplanarity is more difficult to establish within ceiling-like displays, not that ceiling-like spatial arrangements are more generally organized less efficiently than ground-like arrangements. By employing stimulus displays composed of frontoparallel elements (Figure 3), Experiment 2 eliminated differences in local 3-D orientation between ground-like and ceiling-like displays and also provided stimuli in which the multiple rows of elements to be searched were not coplanar (Figure 3). If differences in the 3-D orientation of local stimulus elements in Experiment 1 contributed significantly to the asymmetry observed or if the relative difficulty of performance with ceiling-like displays arose in encoding coplanarity among the subset of elements to be searched, RTs should vary little between the ground-like and the ceiling-like displays of Experiment 2. Conversely, if the inferior performance obtained with ceiling-like displays reflects a more general difficulty in encoding a global arrangement in which objects are placed farther in depth as they are located lower in the field of view, the results of the first experiment should be replicated.

\section{Method}

Observers. The observers were 9 women and 4 men, ages 19-41 years of age. All the participants had normal or corrected-to-normal acuity, normal color vision, and normal stereopsis. Four of these observers had taken part in Experiment 1, whereas the remaining participants were relatively inexperienced psychophysical observers. Three observers were aware of the experimental hypothesis; the remaining observers were naive. Naive observers showed approximately the same magnitude of effect as nonnaive observers.

Apparatus and Stimuli. The apparatus was the same as that used in Experiment 1. The stimuli for Experiment 2 are depicted schematically in Figure 3 . The stimuli were identical to those used in Experiment 1, except that the individual stimulus elements were now frontoparallel. Thus, whereas the displays in Experiment 1 had appeared as vertical stacks of three implicit, horizontally receding planes, the displays in Experiment 2 appeared as vertical stacks of three implicit, horizontally receding strata. Each group of three rows that had appeared as a single implicit plane in Experiment 1 now appeared as a single stratum, with Rows 1, 2, and 4 again forming the top stratum, Rows 3,5, and 7 the middle stratum, and Rows 6,8 , and 9 the bottom stratum.

Procedure. The procedure was similar to that in Experiment 1, but the subset of elements that the observers were to search now formed a horizontal stratum of the larger display, rather than a layer of coplanar elements. The analysis was identical to that in Experiment 1 .

\section{Results and Discussion}

Mean RTs and error rates are presented in Figure 4. Consistent with previous results (He \& Nakayama, 1995), RTs were generally longer for search within the displays in Experiment 2, in which the rows to be searched were noncoplanar, than for search within the displays in Experiment 1 . Of foremost concern, however, RTs for search within ground-like displays showed a mean advantage of $203.22 \mathrm{msec}[S E=76.62 ; F(1,12)=7.04, p=.021]$, 
A.
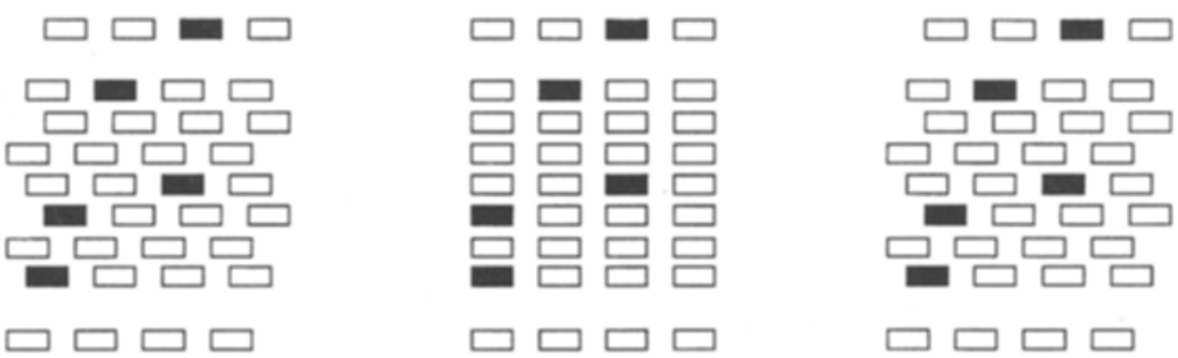

B.
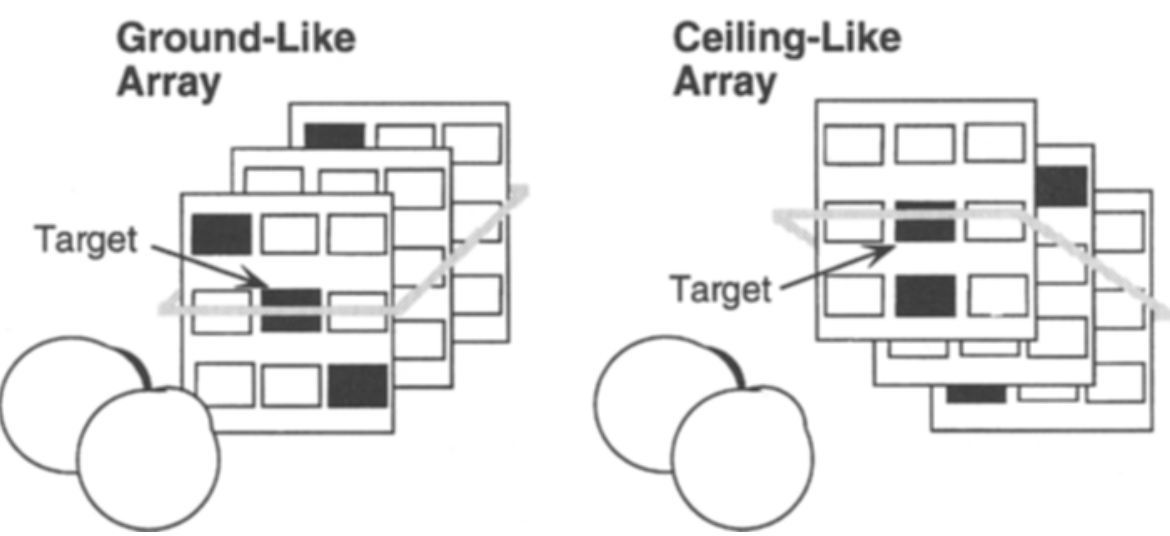

Figure 3. A: stereograms depicting stimuli like those used in Experiment 2. B: a schematic illustration of the stimuli used in Experiment 2.

replicating the primary result of the first experiment and confirming that differences in performance between ground-like and ceiling-like displays are not tied to local orientation of stimulus elements or to coplanarity of the elements to be grouped. RTs demonstrated a reliable tendency to increase with the number of target-colored distractors $[F(2,24)=9.39, p=.001]$, but no less so within ground-like displays than within ceiling-like displays. Thus, the asymmetry between displays of opposite orientations again varied little as the number of target-colored dist actors changed $[F(2,24)=1.42, p=.262]$. As before, RTs were shorter for target-present responses $[F(1,12)=$ $37.12, p<.001]$. No further effects within the RT data reached statistical significance. An analysis of error rates indicated that performance was reliably more accurate with ground-like displays $[F(1,12)=22.08, p=.001]$, precluding the possibility that differences in RTs between ground-like and ceiling-like stimuli represented a speed-accuracy tradeoff. Error rates, as is common, were also higher for target-present than for target-absent trials $[F(1,12)=8.17, p=.014]$. No further effects within the error rate data reached statistical significance.

\section{EXPERIMENT 3}

Experiment 3 was conducted in order to elaborate on the preceding results in the following two ways. First,
Experiment 3 was performed to ensure that the asymmetry observed above did not reflect a bias of performance strategy, rather than a true perceptual anisotropy. Within Experiments 1 and 2, the stimuli varied in orientation unpredictably from trial to trial. Because of this, it is possible that the results of those experiments did not reflect perceptual asymmetries but arose because observers, under conditions of stimulus uncertainty, adopted a strategy or criterion favoring performance within groundlike displays. No differences between RTs to ground-like and ceiling-like stimuli would then have emerged had stimulus orientation been predictable from trial to trial. To test this possibility, Experiment 3 presented trials blocked by 3-D stimulus orientation, providing observers perfect foreknowledge of each display's orientation and allowing them to adjust performance strategy accordingly.

Second, the present experiment was conducted to help to specify whether the perceptual locus of the observed asymmetry lies in the early binocular fusion of stimulus displays or in their subsequent perceptual organization. Past research has clearly demonstrated anisotropies in stereoscopic processing in depth (Fox \& Patterson, 1981) and across the visual field (Breitmeyer, Julesz, \& Kropfl, 1975; Previc, Breitmeyer, \& Weinstein, 1995). In particular, asymmetries have been found favoring far disparities in the upper visual field (UVF) and near disparities in the lower visual field (LVF) (Breitmeyer, et al., 1975; Pre- 

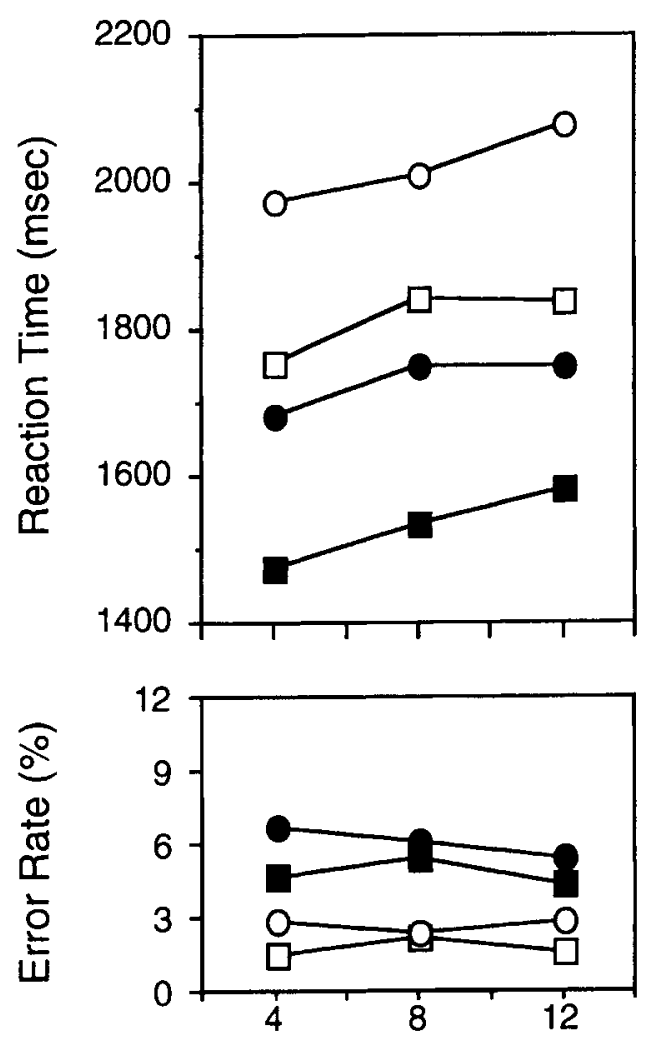

Number of Distractors

$\begin{array}{ll}\text { Target Absent: } & \text { Target Present: } \\ \circ \text { Ceiling-like } & \text { - Ceiling-like } \\ \square \text { Ground-like } & \square \text { Ground-like }\end{array}$

Figure 4. Reaction times and error rates for search of ceilinglike and ground-like displays in Experiment 2, as a function of the number of target-colored distractors within a display.

vic et al., 1995). Breitmeyer et al., measuring the exposure duration necessary for detection of targets presented in depth within dynamic random-dot stereograms, found marked differences in performance across the upper and lower visual hemifields. Against a frontoparallel background plane of crossed disparity, threshold duration was lowest for targets presented in the LVF. Conversely, for targets presented against a background of uncrossed disparity, threshold duration was lowest in the UVF. These effects have since been attributed to the tilt of the vertical horopter, which, as Helmholtz (1910/1962) first realized, does not lie within a plane frontoparallel to the observer's line of sight but is tilted in visual space to extend from the near LVF, through the point of fixation, and into the far UVF (Cogan, 1979; Nakayama, 1977). This tilt places stimuli in the far UVF and near LVF in closer binocular correspondence than stimuli of the same objective dis- parity centered in the near UVF and far LVF (Tyler, 1991) and, Helmholtz suggested, might serve to bring the horopter in line with the ground in front of the observer. Because the ground-like displays within the present experiment extended into the near LVF and far UVF, whereas the ceiling-like displays extended into the far LVF and near UVF, it is possible that longer search times within ceiling-like displays might, in part, reflect longer latencies to stereopsis for stimuli located in the near UVF and far LVF. Previous demonstrations of stereoscopic asymmetries have typically required observers to detect (Breitmeyer et al., 1975) or discriminate (Previc et al., 1995) small test probes of brief duration, defined by their depth within random-dot displays. In contrast, the present experiments, designed to assess 3-D perceptual organization rather than the limits of stereoscopic ability, employed a visual search task and stimuli with clearly visible monocular contours, to allow ready stereoscopic fusion. These differences make it likely that the present results attest to higher level asymmetries, beyond and in addition to anisotropies in local stereopsis. To test this hypothesis directly, Experiment 3 allowed the observers, on some trials, to fixate and fuse achromatic ground-like and ceiling-like configurations before trial onset. At trial onset, then, stimulus elements simply changed color. If the asymmetries demonstrated above were caused entirely by anisotropies in sensory stereoscopic processing, no differences in RTs to ground-like and ceiling-like displays should be apparent when the observers were allowed to achieve fusion before initializing each trial.

\section{Method}

Observers. The observers were 10 men and 1 woman, 2638 years of age. All had normal stereopsis and normal or correctedto-normal acuity. Two observers had taken part either in Experiment 1 or 2 , or in both and in other, similar experiments. All the observers were naive as to the experimental hypotheses.

Apparatus and Stimuli. The apparatus was identical to that in the preceding experiments. The stimuli were identical to those in Experiment 1, except for the following changes. On one half of all the trials, the intertrial interval (ITI) was not filled by a fixation cross but by a ground-like or ceiling-like configuration composed of gray elements. These configurations are hereafter denoted fixation surfaces. The fixation surface preceding a given trial was spatially and stereoscopically identical to that for the trial itself, so that, when the trial began, elements within the gray fixation display changed color but did not change position. The ITI for the other half of the trials was filled by a fixation cross identical to that used in the preceding experiments.

Procedure. The procedure was similar to that in the preceding experiments, except for the following changes. The observers were instructed that, when provided with a 3-D display of gray elements between trials, they should fixate it and use it to prepare themselves for the upcoming trial. The stimuli were blocked by orientation (ground-like or ceiling-like) within larger blocks of fixation type. Each block comprised 11 trials with stimuli of the same orientation and with the same type of fixation display. To ensure that RTs were collected only when the observers were aware of and prepared for the upcoming display's orientation, the 1 st trial of each block was considered a warm-up. Target presence or absence and the number of target-colored distractors were, therefore, determined randomly 

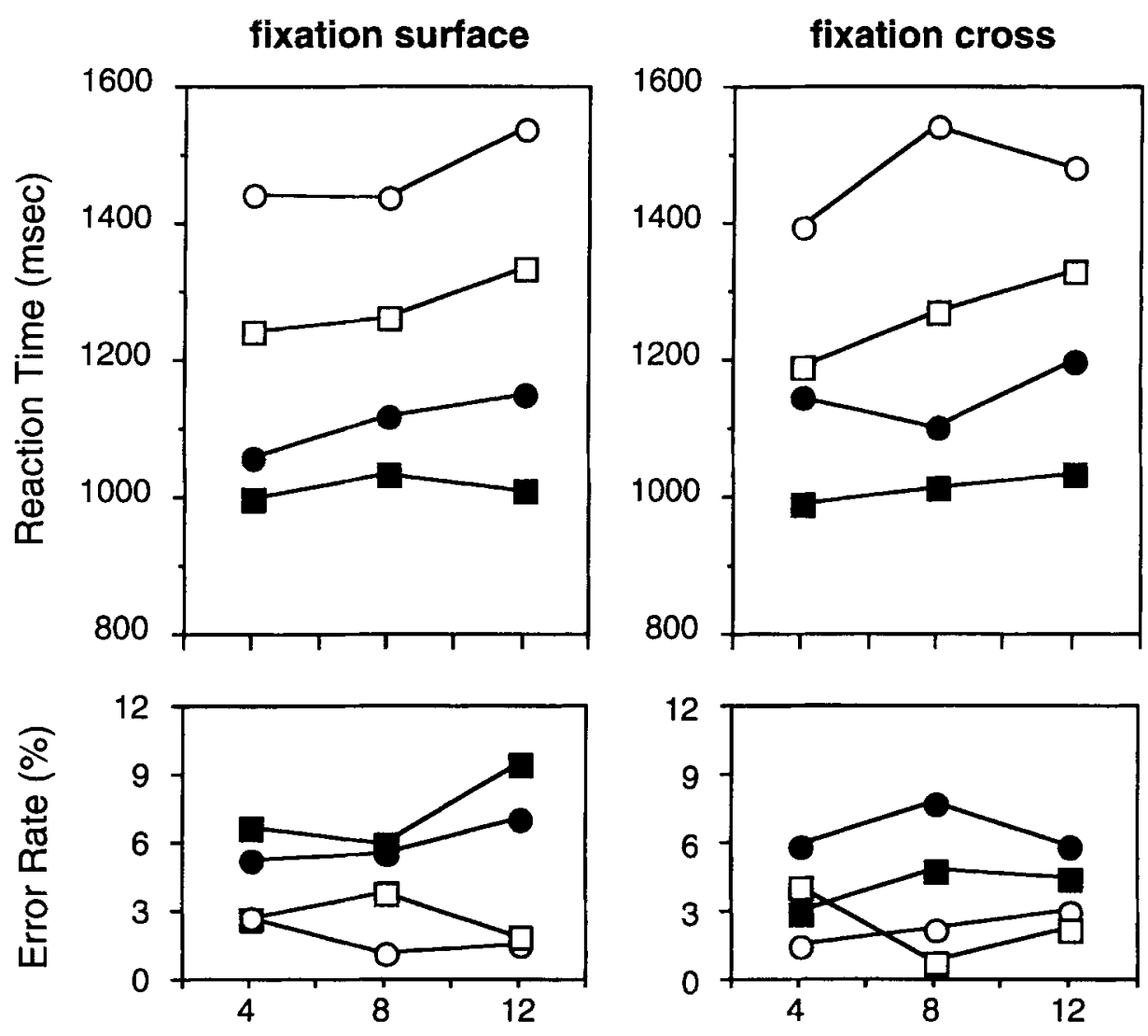

Number of Distractors

\section{Number of Distractors}

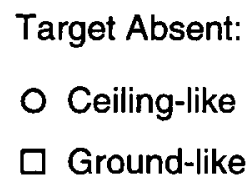

Target Present:

- Ceiling-like

- Ground-like

Figure 5. Reaction times and error rates for search of ceiling-like and ground-like displays preceded by either fixation surfaces or fixation crosses in Experiment 3, as a function of the number of target-colored distractors within a display.

for the 1st trial of each block, and the RT for that trial was disregarded. The order in which the observers completed blocks of trials with fixation crosses and fixation surfaces and the order in which the observers completed blocks of ground-like and ceilinglike trials were counterbalanced. Each observer provided RTs for 25 target-present and 25 target-absent trials at each combination of fixation stimulus, display orientation, and number of target-colored distractors.

\section{Results and Discussion}

Mean RTs and error rates are presented in Figure 5. The data were submitted to a $2 \times 2 \times 2 \times 3$ ANOVA, with fixation condition (fixation surface vs. fixation cross), display orientation (ground-like vs. ceiling-like), target presence or absence, and number of target-colored distractors $(4,8$, or 12$)$ as factors. Several results are noteworthy. First, an asymmetry in RTs was evident once again, favoring performance within ground-like displays by a mean of $157.61 \mathrm{msec}[S E=41.44 ; F(1,10)=14.46$, $p=.003]$, despite the fact that stimuli were blocked by orientation and were preceded on half of all the trials by fixation surfaces. Thus, even with foreknowledge of display orientation, the observers could not perform as efficiently within ceiling-like displays as within ground-like displays. The presentation of a gray fixation surface prior to trial onset, moreover, also did little to either reduce overall RTs $(F<1)$ or erase the asymmetry between RTs to ground-like and ceiling-like stimuli $(F<1)$. That is, the ability to fixate and fuse a stimulus configuration before beginning a trial neither facilitated overall performance nor attenuated the difficulty of performance with ceilinglike displays. Among remaining effects in the RT data, only two were statistically reliable: a main effect of target presence, indicating faster responses to target-present displays $[F(1,10)=12.66, p=.005]$, and an interaction 
of display orientation with target presence $[F(1,10)=$ $14.09, p=.004]$, indicating an exacerbated asymmetry between ground-like and ceiling-like displays for targetabsent responses. Within error rate data, only the main effect of target presence, indicating more accurate responses to target-absent stimuli, reached significance $[F(1,10)=$ $9.2, p=.013]$, suggesting no speed-accuracy tradeoff within the RT data.

Overall, the results indicate that the asymmetrical RTs to ground-like and ceiling-like displays are not the consequence of a simple bias of strategy or criterion that favors ground-like displays. Within the present experiment, the observers were informed of each upcoming display's orientation and could, therefore, adopt a performance strategy and adjust any relevant criteria to optimize performance. Nonetheless, performance was poorer within ceiling-like stimuli. This suggests that the inferior performance obtained with ceiling-like configurations was not the result of a postperceptual bias adopted under conditions of stimulus uncertainty but, rather, represents true difficulty in the perceptual processing of the ceiling-like stimuli. The results, which gave little indication that fixation surfaces reduced the magnitude of asymmetry between ground-like and ceiling-like stimuli, further suggest that the asymmetry observed in the preceding experiments can be attributed, at least in part, to processes beyond early stereoscopic fusion, at a higher level of perceptual organization.

It is, perhaps, notable that the opportunity to view a stimulus configuration before initiating a trial did little to reduce overall RTs. Given that fixation surfaces should have allowed the observers to fuse and perceptually organize displays from the fixation surfaces presented prior to a trial, RTs for displays of either orientation might have been expected to decline. The potential facilitation from previewing stimulus displays might have been offset, in part, by the reduced contrast of stimulus onset that accompanied the presentation of fixation surfaces. Within fixation surface conditions, gray stimulus elements were provided for observers to fixate before initiating each trial. At stimulus onset, these elements merely switched color. Within the fixation cross conditions, in contrast, stimulus elements appeared abruptly against a black background, creating an onset of higher contrast and thereby potentially evoking faster responses (Harwerth \& Levi, 1978). A brief control experiment confirmed this hypothesis. The stimuli and procedure were similar to those in Experiment 3 , except that the observers were asked simply to respond with a keypress as quickly as possible following the onset of colored stimulus elements on each trial. One half of all the trials were again preceded by fixation surfaces, and the remaining trials were again preceded by fixation crosses. Seven observers performed 48 trials with each type of fixation display. The results indicated faster responses following presentation of fixation crosses $(M=337.25 \mathrm{msec}, S E=21.91)$ than following presentation of fixation surfaces $[M=364.59, S E=15.92 ; t(6)=$ $2.75, p=.03$ ]. The potential benefits of fixation surfaces to overall performance in Experiment 3, this suggests, might indeed have been reduced by the attenuated contrast of stimulus onset following presentation of fixation surfaces. Incidentally, the RT difference $(27 \mathrm{msec})$ above is fairly close to the estimated normal binocular fusion time $(30 \mathrm{msec})$ reported by previous researchers (Helmholtz, 1910/1962; Tyler, 1991; Woo, 1974).

Finally, it must be noted that, although the results of Experiment 3 indicate that the asymmetry observed here and in the preceding experiments is not simply the result of longer latency to stereopsis within ceiling-like displays, they do not specify concretely where the asymmetry in fact arises. Presumably, the task used here required the observers not simply to achieve stereopsis with each display, but to perceptually group and segregate a subset of items from within an embedding array of distractors and then to search the isolated subset. Thus, asymmetries in performance that obtained even when the observers were provided with fixation surfaces could have reflected the efficiency with which grouping and selection of relevant items was achieved and maintained, the efficiency of attentional control and search in 3-D space, or both (Andersen, 1990; Andersen \& Kramer, 1993; Atchley, Kramer, Andersen, \& Theeuwes, 1997; He \& Nakayama, 1995; Nakayama \& Silverman, 1986; Theeuwes, Atchley, \& Kramer, 1998). It is possible, for example, that prior to trial onset, the observers perceptually segregated the multiple layers of the fixation surfaces and attended to the layer to be searched but that the onset of colored items within the top and bottom layers of the stimulus display, concomitant with the onset of color within the layer to be searched, captured attention from the relevant items (see, e.g., Nakayama \& Mackeben, 1989; Yantis, 1993). In fact, obligatory capture of 3-D attention by stimulus onset has been reported by Theeuwes et al. (1998). They demonstrated that attentional selection of multiple items within a frontoparallel depth plane can be disrupted by the abrupt onset of an irrelevant stimulus outside of that plane. It is probably to be expected, therefore, that attentional selection of a plane extended in depth, as in the present displays, might likewise be disrupted by the onset of irrelevant stimuli within embedding planes of distractors. Asymmetries in RTs could thus have resulted from asymmetries in the times necessary to regroup and select relevant items within displays of opposite orientations. Alternatively, the present effects might have resulted because ceiling-like displays were attentively searched more slowly following onset of colored stimuli. Further research will be necessary to indicate which of these factors, if not both, is relevant.

\section{GENERAL DISCUSSION}

The organization of the terrestrial observer's visual world is constrained by the ground surface, from which objects in the environment must ultimately, directly or indirectly, find support (Gibson, 1950, 1979). The demand for underlying support ensures that the surfaces and ob- 
jects surrounding the observer are typically arranged to recede in depth with height in the field. The present results demonstrate that the visual system preferentially organizes stimuli distributed in 3-D space to represent surfaces or patterns receding top-away in depth, consistent with this ecological regularity. Items distributed in depth and embedded within a 3-D array of distractors are more easily grouped and searched when they are arranged as if attached to an underlying ground surface, receding with depth in the field, than if they are arranged as if attached to an overhanging ceiling. This phenomenon, importantly, is not predicted by differences within the stimuli themselves. Because the ground-like and ceiling-like arrays of the present experiments were identical in structure, differing only in 3-D orientation, the ground-like arrays contained no inherent physical regularities that could allow them to be more readily or economically encoded than the ceiling-like arrays. Rather, the arrangement of elements within the displays differed most notably in ecological and behavioral relevance; ground-like arrays approximated the spatial relationships most characteristic of the objects and surfaces in the visual environment of humans. Thus, perceptual organization of these displays was guided not only by their internal structure, but also by the spatiotemporal redundancy and behavioral relevance of that structure. Visual stimuli are more easily organized to represent an ecologically pervasive and behaviorally meaningful stimulus pattern - here, the pattern of distal arrangements dictated by the ground surface. This constitutes a lucid example of perception guided by a Helmholtzean likelihood principle (Helmholtz, 1910/1962; Pomerantz \& Kubovy, 1986), and accords with the predictions of Attneave (1954) and Barlow (1961), who proposed that perceptual and neural coding, to avoid impossible inefficiency, must preferentially represent recurring and behaviorally important external patterns.

The application of this principle should also extend beyond the processing of information from binocular disparity. Although the present experiments employed stimuli in which 3-D configuration was given only by stereopsis, the layout of the observer's environment was specified by a variety of static and dynamic cues (Sedgwick, 1986). If, as Experiment 3 suggested, the asymmetry of performance examined here represents an asymmetry in perceptual organization and not a lower level anisotropy in sensory stereopsis, similar effects might be expected in the processing of stimuli in which spatial layout is specified by cues other than binocular disparity. Further research will be necessary to determine whether the present effects are thus generalizable.

It is difficult to specify a neuroanatomical locus for the superior encoding of ground-like layout on the basis of psychophysical data alone, but it is reasonable to speculate that an asymmetry related to the global arrangement of spatially and stereoscopically distributed items has its locus beyond the relatively small receptive fields of V1, perhaps in the extrastriate areas involved in perceptual organization (Merigan, Nealey, \& Maunsell, 1993) or spatial representation (Stein, 1992). It is also interesting to note that the evolutionary onset of Australopithecus was marked by a reorganization of the pongid brain, by which visual and multimodal areas of the extrastriate cortex were increased in size relative to V1 (Holloway, 1995). These changes, occurring 3-4 mya (Holloway, 1995), apparently followed geographical and climatic changes that left the hominoids of East Africa to evolve within an environment drier and less densely wooded than that which had existed there and persisted in West Africa (Coppens, 1995). Holloway suggests that the opportunity for bipedal locomotion within this sparse and open savanna and the accompanying increase in interaction along the ground surface and opportunity for visuomotor manipulation with the newly freed hands rewarded the development of enhanced visuospatial representation, precipitating the cortical reorganization evident in Australopithecus. This, too, may suggest that the superior encoding of spatial relationships consistent with the terrestrial ecology is rooted beyond the striate cortex, in the extrastriate areas that were reorganized so dramatically in response to that ecology.

\section{REFERENCES}

AKS, D. J., \& ENNS, J. T. (1996). Visual search for size is influenced by a background texture. Journal of Experimental Psvchology: Human Perception \& Performance, 22, 1467-1481.

ANDERSEN, G. J. (1990). Focused attention in three-dimensional space. Perception \& Psychophysics, 47, 112-120.

Andersen, G. J., \& Kramer, A. F. (1993). The limits of focused attention in three-dimensional space. Perception \& Psychophysics, 53 , 658-667.

Atchley, P., Kramer, A. F., Andersen, G. J., \& Theeuwes, J. (1997). Spatial cueing in a stereoscopic display: Evidence for a "depthaware" attentional focus. Psychonomic Bulletin \& Review, 4, 524-529.

AtTNEave, F. (1954). Some informational aspects of visual perception. Psychological Review, 61, 183-193.

Barlow, H. B. (1961). Possible principles underlying the transformation of sensory messages. In W. A. Rosenblith (Ed.), Sensory communication (pp. 217-234). Cambridge, MA: MIT Press.

Breitmeyer, B., Julesz, B., \& Kropfl, W. (1975). Dynamic randomdot stereograms reveal up-down anisotropy and left-right isotropy between cortical hemifields. Nature, 187, 269-270.

CoGAN, A. I. (1979). The relationship between the apparent vertical and the vertical horopter. Vision Research, 19, 655-665.

COPPENS, Y. (1995). Brain, locomotion, diet, and culture: How a primate, by chance, became a man. In J. Changeux \& J. Chavaillon (Eds.), Origins of the human brain (pp. 104-112). Oxford: Oxford University Press, Clarendon Press.

DeValors, R. L, \& DeValois, K. K. (1988). Spatial vision. New York: Oxford University Press.

ENNS, J. T., \& RENSINK, R. A. (1990). Influence of scene-based properties on visual search. Science, 247, 712-723.

Fox, R., \& Patrerson, R. (1981). Depth separation and lateral interference. Perception \& Psychophysics, 30, 513-520.

GIBSON, J. J. (1950). The perception of the visual world. Boston: Houghton-Mifflin.

Gibson, J. J. (1979). The ecological approach to visual perception. Hillsdale, NJ: Erlbaum.

Grossberg, S., \& Mingolla, E. (1985). Neural dynamics of form perception: Boundary completion, illusory figures, and neon color spreading. Psychological Review, 92, 173-211.

HARWERTH, R. S., \& LEVI, D. M. (1978). Reaction time as a measure of suprathreshold grating detection. Vision Research, 18, 1579-1586.

HE, Z. J., \& NAKAYAMA, K. (1992). Surfaces versus features in visual search. Nature, 359, 231-233. 
He, Z. J., \& Nakayama, K. (1994a). Apparent motion determined by surface layout not by disparity or 3-dimensional distance. Nature, 367, 173-175.

He, Z. J., \& Nakayama, K. (1994b). Perceiving textures: Beyond filtering. Vision Research, 34, 151-162.

He, Z. J., \& NAKAYAMA, K. (1995). Visual attention to surfaces in threedimensional space. Proceedings of the National Academy of Sciences, 92, $11155-11159$

Helmholtz, H., von (1962). Physiological optics (J. P. C. Southall, Trans.). New York: Dover. (Original work published 1910)

Holloway, R. L. (1995). Toward a synthetic theory of human brain evolution. In J. Changeux \& J. Chavaillon (Eds.), Origins of the human brain (pp. 42-54). Oxford: Oxford University Press, Clarendon Press.

MARR, D. (1982). Vision. New York: Freeman.

Merigan, W. H., Nealey, T. A., \& Maunsell, J. H. R. (1993). Visual effects of lesions of cortical area V2 in macaques. Journal of Neuroscience, 13, 3180-3191.

NakaYama, K. (1977). Geometric and physiological aspects of depth perception. Proceedings of the Society of Photo-Optical Instrument Engineers, 120, 2-9

Nakayama, K., He. Z. J., \& Shimojo, S. (1995). Visual surface representation: A critical link between lower-level and higher-level vision. In S. Kosslyn \& D. O. Osherson (Eds.), An invitation to cognitive science: Vol. 2. Visual cognition (2nd ed., pp. 1-70). Cambridge, MA: MIT Press.

NAKAYAma K., \& MACKEBEN, M. (1989). Sustained and transient components of focal visual attention. Vision Research, 29, 1631-1647.

Nakayama, K., \& Silverman, G. H. (1986). Serial and parallel processing of visual feature conjunctions. Nature, 320, 264-265.

Palmer, S. E., NefF, J., \& Beck, D. (1996). Late influences on perceptual grouping: Amodal completion. Psvchonomic Bulletin \& Review', 3, 75-80

Pomerantz, J. R., \& Kubovy, M. (1986). Theoretical approaches to perceptual organization. In K. R. Boff, L. Kaufman, \& J. P. Thomas (Eds.), Handbook of perception and human performance (pp. 36.136.46). New York: Wiley.

Previc, F. H., Breitmeyer. B. G., \& Weinstein, L. F. (1995). Discriminability of random-dot stereograms in three-dimensional space. International Journal of Neuroscience, $\mathbf{8 0}, 247-253$.

Ramachandran, V. S., \& Cavanagh. P. (1985). Subjective contours capture stereopsis. Nature, 317, 527-530.
Rock, I., \& Brosgole, L. (1964). Grouping based on phenomenal proximity. Journal of Experimental Psychology, 67, 531-538.

SEDGWICK, H. A. (1983). The geometry of spatial layout in pictorial representation. In M. A. Hagen (Ed.), The perception of pictures: Vol. I. Alberti's Window (pp. 425-458). New York: Academic Press.

SEDGwick, H. A. (1986). Space perception. In K. R. Boff, L. Kaufman, \& J. P. Thomas (Eds.), Handbook of perception and human performance (pp. 21.1-21.57). New York: Wiley.

Shimojo, S., \& Nakayama, K. (1990). Amodal presence of partially occluded objects determines apparent motion. Perception, 19, 285299.

STEIN, J. F. (1992). The representation of egocentric space in the posterior parietal cortex. Behavioral \& Brain Sciences, 15, 691-700.

Theeuwes, J., Atchley, P., \& Kramer, A. F. (1998). Attentional control within three-dimensional space. Journal of Experimental Psychology: Human Perception \& Performance, 24, 1476-1485.

Treisman, A., \& Gelade, G. (1980). A feature integration theory of attention. Cognitive Psychology, 12, 97-136.

TyLER, C. W. (1991). The horopter and binocular fusion. In D. Regan (Ed.), Vision and visual dysfunction: Vol. 10. Binocular vision (pp. 19-37). London: Macmillan.

Woo, G. S. (1974). The effect of exposure time on the foveal size of panum's area. Vision Research, 14, 473-480.

YANTIS, S. (1993). Stimulus-driven attentional capture. Current Directions in Psychological Science, 2, 156-161.

\section{NOTE}

1. In the traditional visual search task, the slope of the RT function is often taken as an estimate of the amount of time required to serially process individual stimulus items (e.g., Treisman \& Gelade, 1980). For a variety of reasons, however, this interpretation is inapplicable within the visual search task used here. Effects of target-colored distractors are reported here only as an indication of the general amount of interference produced by heterogeneirty of items outside the relevant layer of the display.

(Manuscript received February 25, 1998; revision accepted for publication March 29, 1999.) 